\title{
Hidden conformations in Aspergillus niger Monoamine Oxidase key for catalytic efficiency
}

\author{
Christian Curado-Carballada, ${ }^{[a]}$ Ferran Feixas, ${ }^{[a]}$ Javier Iglesias-Fernández, ${ }^{[a]}$ and Sílvia Osuna*[a][b] \\ [a] Institut de Química Computacional i Catàlisi (IQCC) and Departament de Química, Carrer Maria Aurèlia Capmany 69, 17003 Girona, Catalonia, Spain \\ E-mail: silvia.osuna@udg.edu \\ [b] ICREA, Pg. Lluís Companys 23, 08010 Barcelona, Spain
}

\begin{abstract}
Enzymes exist as an ensemble of conformational states, whose populations can be shifted by substrate binding, allosteric interactions, but also by introducing mutations to their sequence. Tuning the populations of the enzyme conformational states through mutation enables evolution towards novel activity. In this paper, we use Markov state models to unveil hidden conformational states of Monoamine Oxidase from Aspergillus niger (MAO-N). These hidden conformations, not previously observed by means of any other technique, play a crucial role in substrate binding and enzyme activity. Our work reveals how distal mutations regulate MAO-N activity by stabilizing these hidden catalytically important conformational states, but also by modulating the communication pathway between both MAO-N subunits.
\end{abstract}

The intrinsic dynamism of enzymes plays an essential role in their regulation, inhibition, catalytic activity and promiscuity, but also for their evolvability. ${ }^{[1]}$ The ability of enzymes to acquire new functions has been recently attributed to the many different conformations they adopt in solution other than the native state. This ensemble of conformations that compose the conformational landscape of the enzyme plays an important role in, for example, recognizing and binding the substrate or for releasing the product. The introduction of mutations by natural or laboratory evolution can induce a redistribution on the populations of these existing conformational states and favor novel activities. ${ }^{[1 \mathrm{~b}, 2]}$ This has been shown by analyzing the changes on B-factors of loops along laboratory evolutionary pathways, as well as by extensive MD simulations. ${ }^{[1 \mathrm{c}, 3]}$ Indeed, the engineering of flexible loops that gate the substrate access to the active site and facilitate product release has been shown to be crucial for enhanced catalytic activity. ${ }^{[4]}$

A common feature observed in many Directed Evolution (DE) experiments, is the introduction of remote mutations from the catalytic center, which often have a profound effect in the enzyme catalytic activity. ${ }^{[1 \mathrm{~b}, 5]}$ As it happens in allosterically regulated enzymes, distal mutations regulate the enzyme activity by stabilizing pre-existing catalytically important conformational states. A particularly interesting enzyme in this regard is Monoamine Oxidase from Aspergillus niger (MAO-N), whose catalytic activity and substrate scope has been

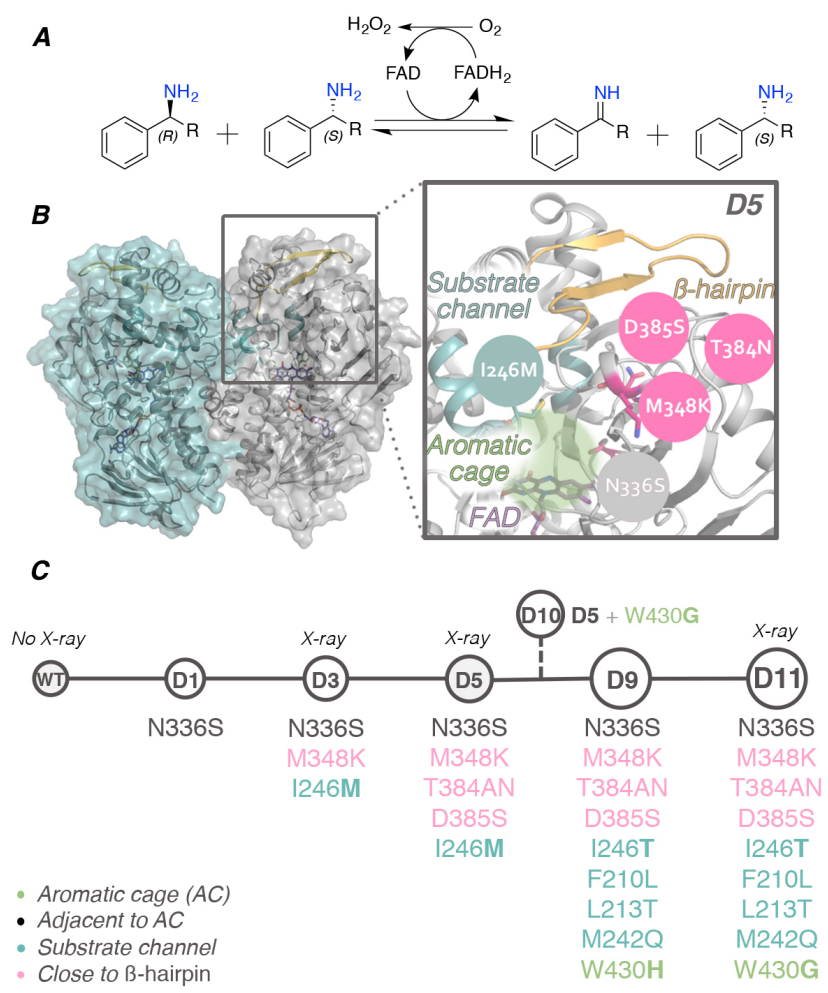

drastically modified by means of both active site and distal mutations (see mutation list in Figure $1 \mathrm{C}$ ). ${ }^{[6]}$ MAO-N features an inner hydrophobic active site cavity located $15 \AA$ from the enzyme surface, where the Flavin Adenine Dinucleotide (FAD) cofactor responsible for the oxidation of amines into the corresponding imines is located (Figure 1A, and 1B). Engineered variants of MAO-N in combination 
with a non-selective chemical reductant has become a powerful strategy for the deracemisation of chiral amine mixtures and, thus, is of interest for obtaining chiral amine building blocks. ${ }^{[6 c]}$

Figure 1. A. MAO-N general reaction scheme, B. Homodimeric structure of MAO-N-D5 where Directed Evolution mutations are highlighted and colored depending on the location with respect to key structural elements: substrate channel (teal), B-hairpin (orange), aromatic cage residues (Phe466 and Trp430, green region), and FAD cofactor in purple. C. Summary of the evolutionary pathways of MAO-N performed by the Turner lab (gray lines). The size of the spheres is indicative of the size of the substrates accepted for each variant. Available X-ray structures are also denoted.

MAO-N wild-type (WT) enzyme, whose X-ray structure has not yet been solved, is only active towards simple straight-chain primary and some secondary amines. ${ }^{[6 a]}$ The first variants that could be successfully crystallized were MAO-N-D3 and MAO-N-D5. ${ }^{[7]}$ Particularly interesting is MAO-N-D5, which was the first variant able of catalyzing the oxidation of different aromatic secondary and chiral tertiary amines with high conversions and enantioselectivities. ${ }^{[6]]}$ Further rounds of evolution yielded MAO-N-D9 and MAO-N-D11 of interest for the production of alkaloid natural products. ${ }^{[6]}$ Interestingly, the mean distance of the introduced mutations with respect to the active site is ca. $18-19 \AA$ in all these DE studies. However, the impact of these mutations on the conformational heterogeneity still remains unclear. This fact combined with the restricted number of available $\mathrm{X}$-ray structures suggests that MAO-N might present an intriguing dynamism of importance for its evolution towards higher activities and bulkier substrates. To evaluate the interplay between conformational dynamics, active site accessibility, and catalytic turnover, we reconstructed by means of extensive long-timescale MD simulations the conformational landscape of MAO-N wild-type and D5 variant. Our results reveal a delicate equilibrium between closed, and hidden partially closed and open states that modulate the enzyme catalytic activity as well as its substrate scope.

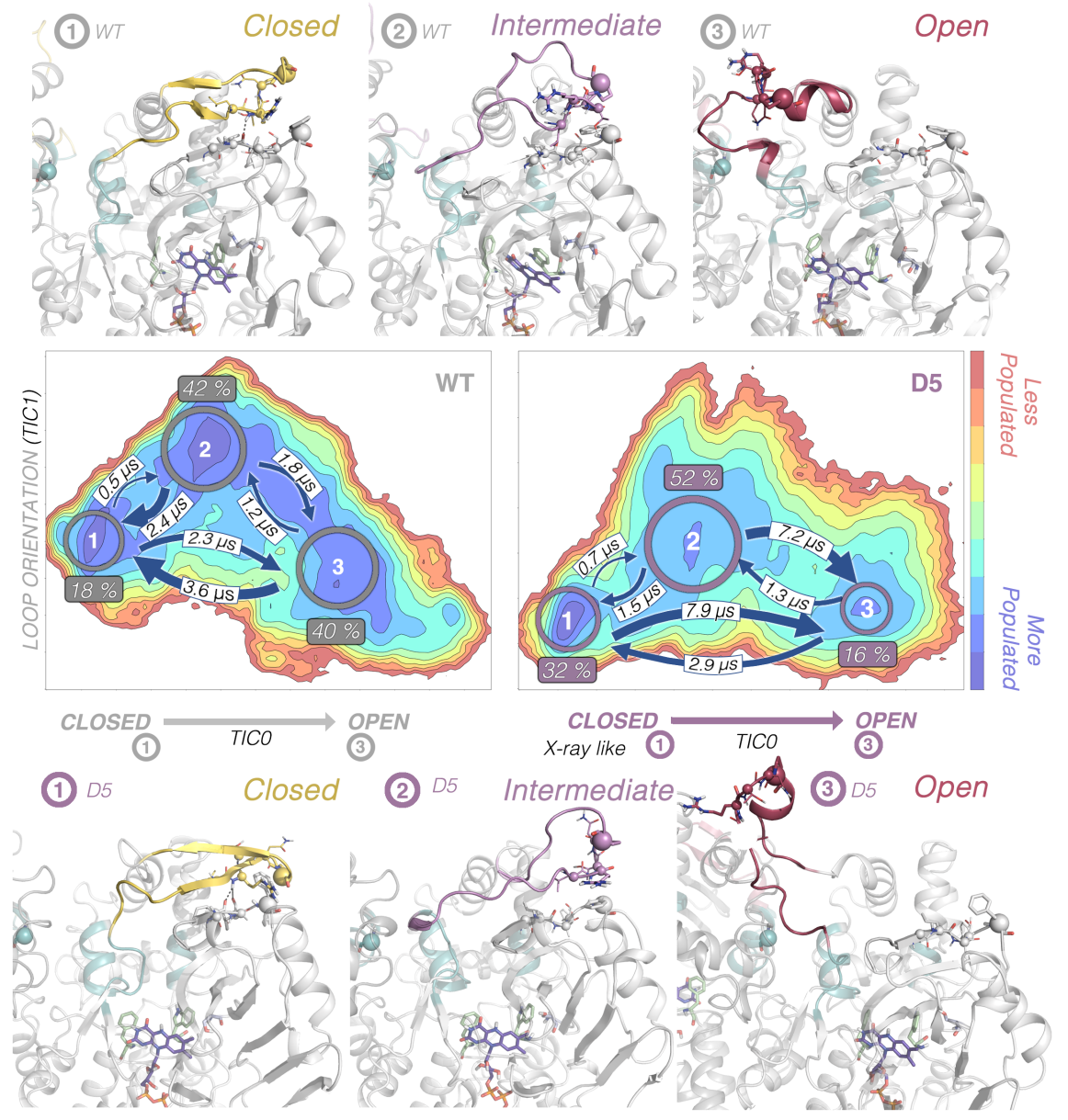

MAO-N conformational dynamics: An initial exploration of the conformational dynamics of MAO-N WT and D5 by means of the unconstrained enhanced sampling technique accelerated Molecular Dynamics (aMD) ${ }^{[8]}$ revealed a highly flexible $ß$-hairpin region formed by residues 124-141 (highlighted in yellow in Figure 1B). These $1 \mu \mathrm{s}$ aMD simulations unveiled two different conformational states, a closed i.e. X-ray like structure, and a hidden open conformational state (Figure 2, states marked as 1 and $\mathbf{3}$, and Figure S7). This observation corresponds to the first evidence of the presence of non-native hidden conformations in MAO-N. Such closed-to-open transition occurs after the loss of the secondary structure of the mentioned ß-hairpin, which is intrinsically disordered when escaping from the closed state. To further characterize the relative stabilities of both states, as well as the associated energy barrier for the opento-closed conformational transition, metadynamics simulations were carried out. ${ }^{[9]}$

Figure 2. Reconstructed Free Energy Landscape (FEL) obtained from an accumulated simulation time of $75 \mu$ s for WT and MAO-N-D5 together with the hidden Markov State Model (hMSM). In hMSM, the relative populations (in \%) of the closed (1 or X-ray like), and the hidden partially closed (2) and open (3) states are 
represented with differently sized spheres. Timescales for the different transitions are indicated with arrows. For each meta-stable state, a representative structure is shown for wild-type (WT, upper part) and D5 (lower), where the B-hairpin is highlighted in either yellow in closed state 1, light pink in partially closed 2, and raspberry in open 3 . The alpha-carbon of the residues that have a higher contribution on TIC0 and TIC1 are also marked with spheres in the WT and D5 structures (see SI)

Proper selection of the required set of collective variables (CVs), describing the open-to-closed conformational transition, was based on the analysis of aMD representative snapshots (see SI for details). Metadynamics simulations indicate that the closed state is highly favored in D5 compared to WT, and that the associated energy barrier required for the closed-to-open conformational transition is ca. 4 and $11 \mathrm{kcal} / \mathrm{mol}$ for WT and D5, respectively (see Figure S1). The stabilization of the closed state in the evolved variant suggests that a population shift occurs and that WT and D5 variants can present different kinetics for the closed-to-open transition.

To completely characterize the intricate nature of this relevant transition, Markov state models were used. ${ }^{[10]}$ In particular, a hidden Markov State Model (hMSM) ${ }^{[11]}$ was built with the accumulated $75 \mu$ s of conventional Molecular Dynamics (MD) simulations for each variant (i.e. $150 \mu \mathrm{s}$ in total, see Figure 2 and full details in SI and Figures S3-S5). In hMSM the conformational dynamics of WT and MAO-N D5 were unveiled and coarse-grained into three main conformational meta-stable states: closed (1 in Figure 2), and the hidden partially closed (2) and open (3).

MAO-N WT enzyme is able to explore a vast number of equally stable conformations highly populating the hidden disordered partially closed (2), and many open states (3). The computed hMSM indicates that the closed state has a relative population of $19 \%$, whereas hidden partially closed and open states are the most stable conformations (populations of $42 \%$ and $39 \%$, respectively). The kinetics of the conformational state interconversion indicates that the transition from closed to partially closed states is quite fast (ca. $600 \mathrm{~ns}$ ), however, escaping from partially closed and open meta-stable states has a substantially higher energetic penalty (a timescale of ca. 4 us for the open-to-closed transition). These timescales are in line with the energy barriers observed in metadynamics. As shown in Figure 2, partially closed and open states are characterized by the disruption of the backbone hydrogen bonding network between residues located at the extremes of the ß-hairpin region. The large number of thermally accessible conformational states that present a disordered $ß$-hairpin loop might be the main reason behind the difficulties encountered in MAO-N WT X-ray characterization.

Interestingly, the introduced mutations in D5 variant induce a population shift toward closed and partially closed conformations (the relative populations are now $32 \%$ and $52 \%$, respectively), being open states the lowest populated ones (16\%). The timescale associated to the closed-to-open transition is ca. $8 \mu \mathrm{s}$, which is substantially higher than in WT (ca. $2 \mu \mathrm{s}$ ). Additionally, the transition from partially closed to open states is ca. $7 \mu \mathrm{s}$ (in WT is ca. $2 \mu \mathrm{s}$ ), indicating that open states in D5 are both thermodynamically and kinetically disfavored. Interestingly, the interconversion from closed to partially closed states is quite fast (ca. $700 \mathrm{~ns}$ ), and also the back transition from partially closed to closed states $(1.5 \mu \mathrm{s})$. It is worth mentioning that the different populations and kinetics obtained suggest that the excellent performance of D5 towards the oxidation of amines arises from the stabilization of the closed and the hidden partially closed conformations.

Interplay between conformational dynamics and catalysis: After the extensive evaluation of the rich conformational landscape of MAO$\mathrm{N}$, two questions remain to be answered: (1) how important is the ß-hairpin conformational dynamics for catalysis? And (2) why MAON WT is active mainly towards aliphatic primary amines (for instance hexylamine, HEX), whereas D5 shows improved activity towards bulkier substrates such as alpha-methyl benzylamine (AMBA)? To explore the interplay between the conformational features of MAO$\mathrm{N}$ variants and its different substrate preferences, we studied the molecular basis of the substrate binding process. Initial evaluation of substrate access channels on the different energy minima with the CAVER software highlighted two main entry routes close to the $B$ hairpin for both WT and D5 systems (Figure S6 and S12). As found in previous studies, ${ }^{[7]}$ channel 1 starting at the hinge region of the ß-hairpin was identified, as well as a second channel (channel 2, see Figure 3 and S6) that could also play a role in substrate binding. To decipher the key steps of the binding mechanism of WT and D5, we performed free substrate binding simulations with aMD, starting from representative closed and hidden open conformations (see Figure 3 and Figures S8-S10).

In both WT and D5 variants, binding only occurs in those monomers displaying closed or the hidden partially closed state (see Figure 2 and S2). Interestingly, it is also a requisite for binding that the other monomer adopts either an open or partially closed conformation, thus pointing out cooperative motions between the two subunits. Substrate binding is not observed when both monomers display a closed conformation indicating that the ß-hairpin conformational dynamics is essential for substrate recognition and binding. For WT, the recognition of HEX substrate takes place at the dimer interface near Glu145 and Arg123 residues located at the hinge of the $B-$ hairpin (see yellow residues in Figure 3 and Figure S9). The substrate enters channel 1 of monomer B when the B-hairpin stays in a closed conformation and monomer A exhibits a partially closed state. The aliphatic chain of the substrate establishes hydrophobic interactions with the side-chains of Met242 and lle246 located at the channel entrance (see binding event 1-WT in Figure 3A). When the salt bridge between Glu145 and Arg123 is broken (distances over $8 \AA$ ), the substrate is allowed to access the active site tunnel (see 2 in Figure $3 A$ and S7). Once inside channel 1, access of HEX towards the aromatic pocket where the FAD cofactor is located is blocked by Trp94 and Leu213. After some simulation time, the nearby Phe382 undergoes a conformational change providing extra space for Leu213 and Trp94 to change the side-chain conformation. This allows the substrate to completely access the active site and establish catalytically competent distances with the FAD. Catalytically competent distances between HEX and FAD are observed irrespective of the orientation of the aromatic cage residues Phe466 and Trp430. These simulations indicate that Phe382, Leu213 and Trp94 regulate HEX-binding in WT. 

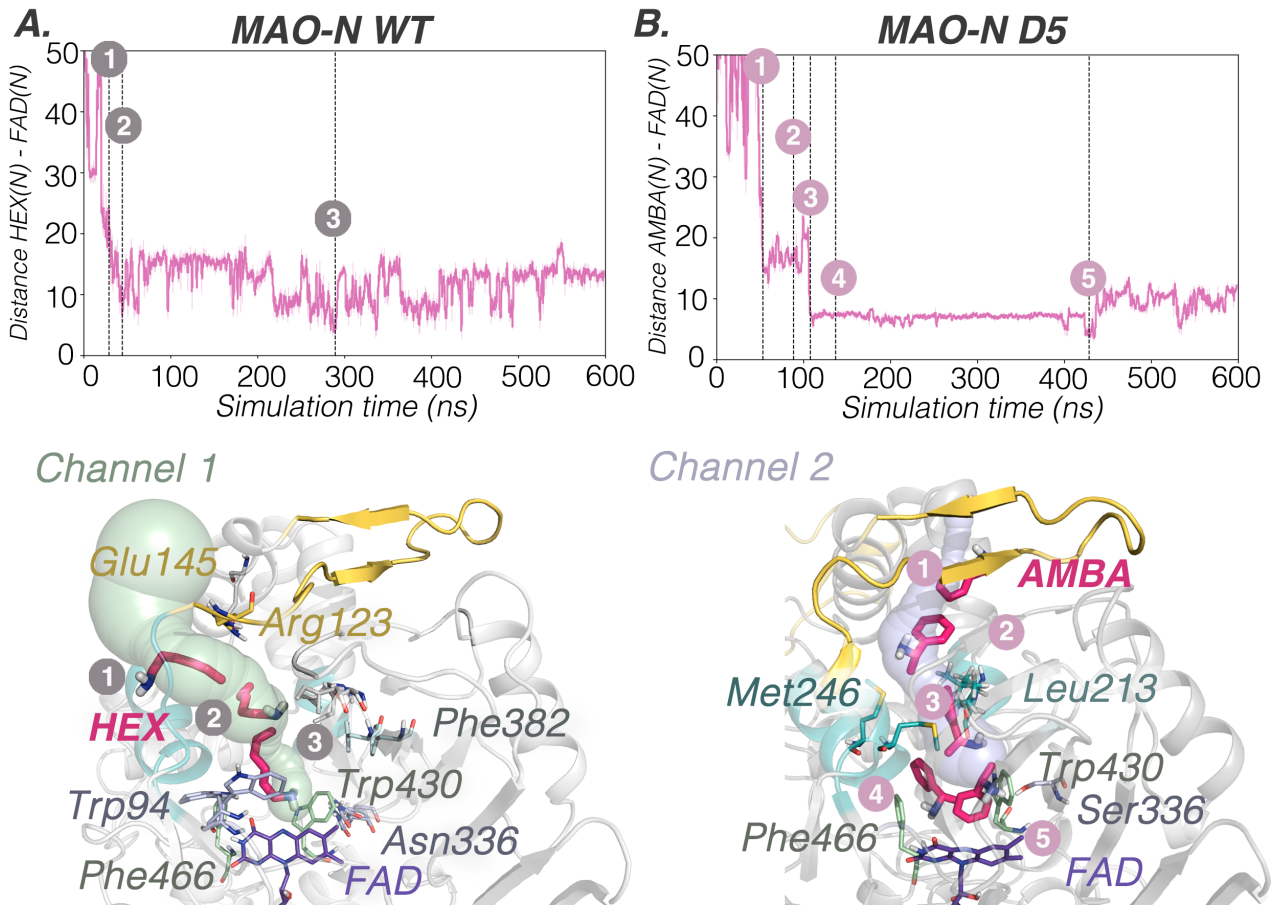

Figure 3. Plot of the distance between one of the representative 600 ns aMD replicas between the FAD cofactor and A) hexylamine (HEX) or B) alpha-methyl benzylamine (AMBA). Representative snapshots taken at different points along the simulation (see numbers in plot and structure) of the reconstructed binding pathway are shown for both WT and D5 enzymes. Substrate access channels 1 and 2 are also represented in green and blue, respectively.

The reconstructed binding pathway for the bulkier AMBA and D5 variant indicates that the mutations introduced alter the conformational dynamics of the enzyme, but also its binding mechanism. The recognition is assisted by the side-chains of monomer A $B$-hairpin hydrophobic residues (see recognition events 1 and $\mathbf{2}$ in Figure 3B and Figure S10). At this point, monomer $A$ is found in a closed state, whereas monomer $B$ is in an open/partially closed conformation. Prior to AMBA recognition, the B-hairpin of monomer A performs a lateral displacement that resembles the one observed in the partially closed state. The recognition occurs through channel 2 rather than 1 observed in WT (see Figure 3). After recognition, AMBA rapidly progresses through the substrate channel where the new Met246 introduced (i.e. lle246Met) acts now as a gate and modulates the access to the aromatic cage ( 3 in Figure 3B). At some point, Met246 undergoes a side-chain conformational change that allows AMBA to get closer to the active site aromatic cavity in a non-productive conformation (4 in Figure 3B, distances between $\mathrm{AMBA}(\mathrm{N})$ and $\mathrm{FAD}(\mathrm{N})$ longer than $6 \AA$ ). After 300 ns of aMD simulation time, AMBA gets properly positioned between the aromatic cage residues Phe466 and Trp430 establishing m-stacking interactions with both residues. This corresponds to the catalytically competent pose (with distances between FAD and AMBA shorter than ca. $4 \AA$ ), which is maintained for a few ns in this aMD simulation. In contrast, non-catalytically competent conformations of the aromatic cage were found in WT due to the lower stability of the ß-hairpin, thus explaining the lower activity of WT towards bulkier and rigid substrates such as AMBA.

Interestingly, the repositioning of AMBA in a catalytically productive pose inside the aromatic cage (from $\mathbf{4}$ to $\mathbf{5}$ in Figure 3 ) in D5 is correlated to a partial closing of the ß-hairpin of the other monomer, indicating some cooperativity between the two subunits. The cooperativity between both subunits is further confirmed by our new recently developed Shortest Path Map (SPM) tool (see Figure S11). ${ }^{[3 b]}$ The mutations introduced along DE are interestingly located adjacent to the WT SPM path, thus establishing that along the course of evolution the communication between both subunits is altered. Particularly relevant in the WT SPM communication path is Phe466, which forms the aromatic cage of the active site, and whose conformational dynamics is linked to the ß-hairpin motions. Interestingly, the SPM of MAO-N-D5 predicts positions located close to the mutation points introduced in further DE studies for MAO$\mathrm{N}-\mathrm{D} 9$ and D11 generation (see Figure S11). This study further reinforces the suitability of our SPM tool, ${ }^{[3 b]}$ which evaluates the intrinsic conformational dynamics of the target enzyme, for the construction of smart focused libraries for enzyme design.

Our work demonstrates that distal mutations introduced via DE regulate MAO-N activity and substrate scope by modulating the relative stabilities of hidden catalytically important conformational states. The delicate equilibrium existing between these closed, and the hidden partially closed and open states regulates MAO-N catalytic activity by allowing the substrate to reach the active site pocket, but also by altering the active site cavity for efficient catalysis. Our study highlights the key role played by the enzyme conformational dynamics, and evidences that future computational enzyme design strategies will need to further characterize the conformational landscape of enzymes for achieving highly proficient enzymes. 


\section{Experimental Section}

The systems were prepared for Molecular dynamics (MD) simulations using the Leap module from Amber16. The force field used was ff99SBildn utilizing the TIP3P water model. The free energy landscape (FEL) of MAO-N WT and D5 was reconstructed from the accumulation of $75 \mu$ s of conventional MD) simulations for each variant (i.e. $150 \mu$ s in total) with pyEMMA. ${ }^{12]}$ The high dimensional MD data was reduced using the kinetically-relevant time-lagged independent component analysis (TICA) considering the main distances and angles of the highly flexible ß-hairpin (see complete description in SI).

\section{Acknowledgements}

F.F. and J.I.F. thank the European Community for Marie Curie fellowships MSCA-IF-2014-EF-661160, and H2020-MSCA-IF-2016-753045, respectively. S. O. thanks the Spanish MINECO CTQ2014-59212-P, Ramón y Cajal contract (RYC-2014-16846), the European Community for CIG project (PCIG14GA-2013-630978), and the funding from the European Research Council (ERC) under the European Union's Horizon 2020 research and innovation programme (ERC-2015-StG-679001). We thank the Generalitat de Catalunya for grup emergent 2017 SGR-1707. We are grateful for the computer resources, technical expertise, and assistance provided by the Barcelona Supercomputing Center - Centro Nacional de Supercomputación. We thank Dr. Olsson for fruitful discussions and advice on ß-hairpin featurization.

Keywords: Molecular dynamics • distal mutations $\bullet$ enzyme catalysis $\bullet$ monoamine oxidase $\bullet$ substrate binding

[1] a) N. Tokuriki, D. S. Tawfik, Science 2009, 324, 203-207; b) M. A. Maria-Solano, E. Serrano-Hervás, A. Romero-Rivera, J. IglesiasFernández, S. Osuna, Chem. Commun. 2018, 54, 6622-6634; c) E. C. Campbell, G. J. Correy, P. D. Mabbitt, A. M. Buckle, N. Tokuriki, C. J. Jackson, Curr. Opin. Struct. Biol. 2018, 50, 49-57.

[2] a) E. Campbell, M. Kaltenbach, G. J. Correy, P. D. Carr, B. T. Porebski, E. K. Livingstone, L. Afriat-Jurnou, A. M. Buckle, M. Weik, F. Hollfelder, N. Tokuriki, C. J. Jackson, Nat. Chem. Biol. 2016, 12, 944-950; b) D. D. Boehr, R. Nussinov, P. E. Wright, Nat. Chem. Biol. 2009, 5, 789-796.

[3] a) S. Osuna, G. Jiménez-Osés, E. L. Noey, K. N. Houk, Acc. Chem. Res. 2015, 48, 1080-1089; b) A. Romero-Rivera, M. Garcia-Borràs, S. Osuna, ACS Catal. 2017, 7, 8524-8532; c) G. Jiménez-Osés, S. Osuna, X. Gao, M. R. Sawaya, L. Gilson, S. J. Collier, G. W. Huisman, T. O. Yeates, Y. Tang, K. N. Houk, Nat. Chem. Biol. 2014, 10, 431-436.

[4] a) A. Gora, J. Brezovsky, J. Damborsky, Chem. Rev. 2013, 113, 5871-5923; b) N. Kreß, J. M. Halder, L. R. Rapp, B. Hauer, Curr. Opin. Chem. Biol. 2018, 47, 109-116.

[5] A. Currin, N. Swainston, P. J. Day, D. B. Kell, Chem. Soc. Rev. 2015, 44, 1172-1239.

[6] a) R. Carr, M. Alexeeva, A. Enright, T. S. C. Eve, M. J. Dawson, N. J. Turner, Angew. Chem. Int. Ed. 2003, 42, 4807-4810; b) D. Ghislieri, A. P. Green, M. Pontini, S. C. Willies, I. Rowles, A. Frank, G. Grogan, N. J. Turner, J. Am. Chem. Soc. 2013, 135, 10863-10869; c) S. Herter, F. Medina, S. Wagschal, C. Benhaïm, F. Leipold, N. J. Turner, Bioorganic Med. Chem 2017, 30391-30397; d) E. O'Reilly, C. Iglesias, D. Ghislieri, J. Hopwood, J. L. Galman, R. C. Lloyd, N. J. Turner, Angew. Chem. Int. Ed. 2014, 53, 2447-2450; e) G. Li, P. Yao, R. Gong, J. Li, P. Liu, R. Lonsdale, Q. Wu, J. Lin, D. Zhu, M. T. Reetz, Chem. Sci. 2017, 8, 4093-4099; f) C. J. Dunsmore, R. Carr, T. Fleming, N. J. Turner, J. Am. Chem. Soc. 2006, 128, 2224-2225.

[7] K. E. Atkin, R. Reiss, V. Koehler, K. R. Bailey, S. Hart, J. P. Turkenburg, N. J. Turner, A. M. Brzozowski, G. Grogan, J. Mol. Biol. 2008, 384, 1218-1231.

[8] a) Y. Miao, F. Feixas, C. Eun, J. A. McCammon, J. Comput. Chem. 2015, 36, 1536-1549; b) D. Hamelberg, J. Mongan, J. A. McCammon, J. Chem. Phys. 2004, 120, 11919-11929.

[9] A. Laio, M. Parrinello, Proc. Natl. Acad. Sci. USA 2002, 99, 12562-12566.

[10] J. D. Chodera, F. Noé, Curr. Opin. Struct. Biol. 2014, 25, 135-144.

[11] F. Noé, H. Wu, J.-H. Prinz, N. Plattner, J. Chem. Phys. 2013, 139, 184114

[12] M. K. Scherer, B. Trendelkamp-Schroer, F. Paul, G. Pérez-Hernández, M. Hoffmann, N. Plattner, C. Wehmeyer, J.-H. Prinz, F. Noé, J. Chem. Theor. Comput. 2015, 11, 5525-5542. 
